\title{
Modélisation magnétique et numérique par éléments finis de feuilletages ferromagnétiques
}

\author{
J. P. Bastos, C. Jablon, G. Quichaud et F. Rioux-Damidau \\ Laboratoire d'Electrotechnique, Bât. 214, 91405 Orsay, France \\ (Reçu le 16 juillet 1980, révisé le 11 septembre 1980, accepté le 17 septembre 1980)
}

\begin{abstract}
Résumé. - Les circuits magnétiques constitués par des empilements de tôles de fer sont fréquemment utilisés en électrotechnique. Nous proposons ici une modélisation bidimensionnelle de telles structures associée à une méthode variationnelle par éléments finis adaptée aux problèmes pour lesquels il faut tenir compte à la fois de l'anisotropie et de la saturation magnétique.

Abstract. - Stacked iron sheets magnetics circuits are frequently used in electrical engines, but the calculation of the field distribution is difficult by the anisotropy in the iron. We propose in this paper a bidimensional modelisation of these structures using a finite elements variational method which is adapted to such anisotropic saturable magnetic problems.
\end{abstract}

1. Introduction. - L'anisotropie magnétique correspond à la non-coïncidence directionnelle du champ magnétique $\mathbf{H}$ et de l'induction $\mathbf{B}$. Cette anisotropie se trouve dans presque toutes les structures électrotechniques, car elles comportent des tôles feuilletées, mais en général les lignes d'induction sont parallèles au feuilletage et l'anisotropie ne joue pratiquement pas de rôle. Cependant, cette anisotropie doit absolument être prise en compte dans certains cas tels que les effets d'extrémités d'une machine tournante, les machines à réluctance variable polydiscoïdes [3], à feuilletage axial [1], etc...

Quelques modélisations ont été publiées tenant compte de l'anisotropie seule [1, 2,11], ou bien simultanément de l'anisotropie et de la saturation $[12,13]$.

Nous proposons ici une modélisation bidimensionnelle des tôles feuilletées prenant en compte les deux effets. Cette nouvelle méthode fait intervenir un tenseur de perméabilité déterminé à partir du champ magnétique moyen. Ensuite, pour calculer la distribution du champ magnétique dans les tôles, nous utilisons une méthode variationnelle par éléments finis, connue pour son efficacité et sa souplesse [10]. Notons que cette méthode s'applique à tout problème anisotrope bidimensionnel, lorsque l'on peut définir un tenseur de perméabilité.

Nous avons calculé précédemment $[3,5]$ les performances théoriques d'une machine à hautes performances de conception particulière $[4,9]:$ la machine à réluctance variable polydiscoïde. Nous avons utilisé, dans sa région utile (plots), du fer magnétiquement isotrope. Une étape ultérieure consiste à évaluer à l'aide de la méthode évoquée ci-dessus, les performances de cette machine lorsque nous introduisons une anisotropie magnétique dans les plots, nous donnons des exemples de calcul de champ dans ce cas.

2. Modélisation bidimensionnelle d'un feuilletage magnétique. - Supposons que nous ayons le feuilletage régulier représenté sur la figure 1 où la répartition fer-air est ainsi définie :

$e \quad$ : épaisseur totale d'une lame d'air et d'une tôle magnétique

$\varepsilon \quad$ : épaisseur relative d'air

$(1-\varepsilon)$ : épaisseur relative de fer (coefficient de remplissage).

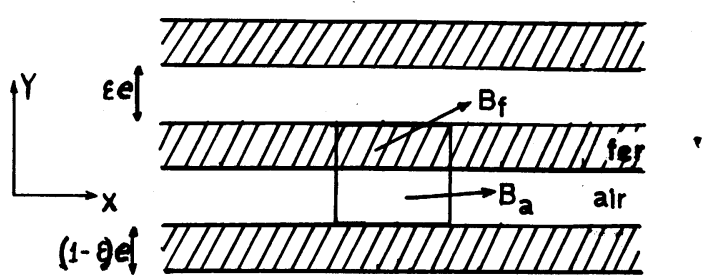

Fig. 1. - Représentation d'un feuilletage fer-air.

[Stacked iron sheets representation.] 
L'épaisseur $e$ étant supposée suffisamment petite, nous désignerons en un point quelconque par $\mathbf{H}_{\mathrm{f}}$ et $\mathbf{H}_{\mathrm{a}}$ les moyennes respectives des champs magnétiques dans les tôles et dans les lames d'air; $\mathbf{B}_{\mathrm{f}}$ et $\mathbf{B}_{\mathrm{a}}$ étant les moyennes des inductions correspondantes nous pouvons alors définir une induction et un champ moyens macroscopiques :

$$
\begin{aligned}
& \mathbf{B}=\varepsilon \mathbf{B}_{\mathrm{a}}+(1-\varepsilon) \mathbf{B}_{\mathrm{f}} \\
& \mathbf{H}=\varepsilon \mathbf{H}_{\mathrm{a}}+(1-\varepsilon) \mathbf{H}_{\mathbf{f}} .
\end{aligned}
$$

Ils vérifient les équations de Maxwell suivantes, où $\mathbf{J}$ représente, le cas échéant, les courants :

$$
\begin{aligned}
\operatorname{rot} \mathbf{H} & =\mathbf{J} \\
\operatorname{div} \mathbf{B} & =0
\end{aligned}
$$

puisque ces vecteurs moyens introduits conservent la circulation et le flux.

La conservation de la composante tangentielle du champ et de la composante normale de l'induction à l'interface fer-air nous permet d'écrire :

$$
\begin{aligned}
& H_{X}=H_{\mathrm{a} X}=H_{\mathrm{f} X} \\
& B_{Y}=B_{\mathrm{a} Y}=B_{\mathrm{f} Y}
\end{aligned}
$$

( $X$ et $Y$ représentent les axes principaux du feuilletage, cf. Fig. 1).

L'expression (2) appliquée à la direction " $Y$ » donne, en tenant compte de (6)

$$
H_{Y}=\left(\frac{\varepsilon}{\mu_{\mathrm{a}}}+\frac{1-\varepsilon}{\mu_{\mathrm{f}}}\right) B_{Y}
$$

et d'une façon analogue avec (1) et (5)

$$
B_{X}=\left(\varepsilon \mu_{\mathrm{a}}+(1-\varepsilon) \mu_{\mathrm{f}}\right) \boldsymbol{H}_{X} .
$$

Ces expressions nous permettent de définir les perméabilités moyennes $\mu_{X}$ et $\mu_{Y}$ telles que :

$$
\begin{gathered}
\frac{1}{\mu_{Y}}=\frac{\varepsilon}{\mu_{\mathrm{a}}}+\frac{1-\varepsilon}{\mu_{\mathrm{f}}} . \\
\mu_{X}=\varepsilon \mu_{\mathrm{a}}+(1-\varepsilon) \mu_{\mathrm{f}} .
\end{gathered}
$$

Mais $\mu_{\mathrm{f}}$ est une fonction $P$ du module de $H_{\mathrm{f}}$ que nous notons

$$
\mu_{\mathrm{f}}=\mu_{\mathrm{a}} P\left(H_{\mathrm{f}}^{2}\right) \quad \text { où } \quad H_{\mathrm{f}}^{2}=H_{\mathrm{f} Y}^{2}+H_{\mathrm{f} X}^{2} .
$$

La fonction de perméabilité $P$ est déduite de la courbe d'aimantation $B(H)$ du fer supposé sans hystérésis.

De la relation (5) on déduit que $H_{\mathrm{f} X}=H_{X}$ et de la relation (6) que :

$$
H_{\mathrm{f} Y}=\frac{H_{Y}}{\frac{\mu_{\mathrm{f}} \varepsilon}{\mu_{\mathrm{a}}}+1-\varepsilon}
$$

$\mu_{\mathrm{f}}$ est donc définie à partir de $\mathbf{H}$ au moyen de l'équation implicite suivante :

$$
\frac{\mu_{\mathrm{f}}}{\mu_{\mathrm{a}}}=P\left(H_{X}^{2}+\frac{H_{Y}^{2}}{\left(\frac{\mu_{\mathrm{f}} \varepsilon}{\mu_{\mathrm{a}}}+(1-\varepsilon)\right)^{2}}\right)
$$

que l'on peut résoudre par approximations successives, comme on le verra par la suite.

3. Le tenseur de perméabilité $[\mu]$. - Nous définissons les perméabilités du matériau anisotrope par $B_{X}=\mu_{X} H_{X}$ et $B_{Y}=\mu_{Y} H_{Y}$ où $X$ et $Y$ sont les axes principaux du matériau. Pour généraliser davantage la méthode, on peut considérer le cas où les axes du matériau font un angle $\theta$ avec les axes de référence $(\mathrm{O} x, \mathrm{O} y)$. Par exemple un matériau constitué par un feuilletage fer-air vertical peut être considéré comme un autre à feuilletage horizontal, tourné de $90^{\circ}$.

Soit alors, sur la figure $2, \mathrm{O} x$ et $\mathrm{O} y$ les axes de référence; $\mathrm{O} X$ et $\mathrm{O} Y$ les axes principaux du matériau, et $\theta$ l'angle entre $O X$ et $O x$.

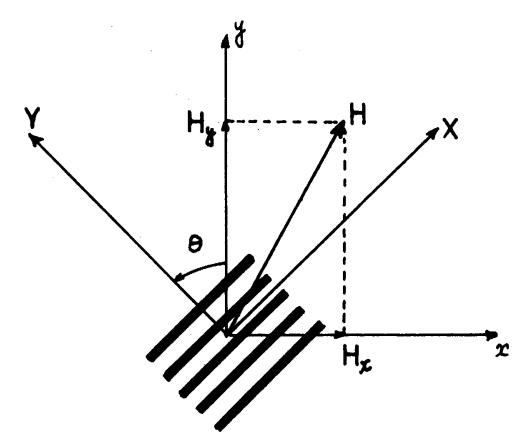

Fig. 2. - Rotation d'axes appliquée au feuilletage.

[Sheets axes rotation.]

Le changement d'axe associé à la matrice de rotation :

$$
[R]=\left[\begin{array}{ll}
\cos \theta & \sin \theta \\
-\sin \theta & \cos \theta
\end{array}\right]
$$

permet ainsi d'écrire :

$$
\left[\begin{array}{l}
B_{x} \\
B_{y}
\end{array}\right]=[R]^{-1}\left[\begin{array}{ll}
\mu_{X} & 0 \\
0 & \mu_{Y}
\end{array}\right][R]\left[\begin{array}{l}
H_{x} \\
H_{y}
\end{array}\right]
$$

soit :

$$
\left[\begin{array}{l}
B_{x} \\
B_{y}
\end{array}\right]=[\mu]\left[\begin{array}{l}
H_{x} \\
H_{y}
\end{array}\right]
$$

ce qui nous donne la matrice de perméabilité suivante :

$$
[\mu]=\left[\begin{array}{ll}
\mu_{x} & \mu_{x y} \\
\mu_{y x} & \mu_{y}
\end{array}\right]
$$


où

$$
\begin{aligned}
& \mu_{x}=\mu_{X} \cos ^{2} \theta+\mu_{Y} \sin ^{2} \theta \\
& \mu_{y}=\mu_{X} \sin ^{2} \theta+\mu_{Y} \cos ^{2} \theta \\
& \mu_{y x}=\left(\mu_{X}-\mu_{Y}\right) \sin \theta \cos \theta=\mu_{x y} .
\end{aligned}
$$

On voit sur les expressions ci-dessus que le cas isotrope se présente comme un cas anisotrope tel que:

$$
\mu_{x}=\mu_{y} \text { et } \mu_{y x}=0 .
$$

La méthode d'éléments finis que nous utilisons pour calculer la distribution du champ magnétique impose le calcul du tenseur de perméabilité $[\mu]$ à partir des champs moyens $H_{x}$ et $H_{y}$, ce qui nécessite la résolution de l'équation implicite (13).

Ainsi, après avoir obtenu $H_{X}$ et $H_{Y}$ à partir de $H_{x}$ et $H_{y}$ au moyen du changement de repère associé à la rotation $[R]$, nous devons résoudre l'équation implicite (13), pour cela nous avons utilisé l'algorithme suivant :

a) on part de la valeur initiale de $H_{\mathrm{f}}=0$ (régime linéaire)

b) avec cette valeur approchée de $H_{\mathrm{f}}$, on calcule $\mu_{\mathrm{f}}$ au moyen de (11)

c) avec $\mu_{\mathrm{f}}$ et $\varepsilon$ on calcule $\mu_{Y}$ au moyen de (9)

d) on calcule $H_{\mathrm{f} Y}$ au moyen de (12)

e) on calcule de nouveau $H_{\mathrm{f}}=\sqrt{H_{X}^{2}+H_{\mathrm{fY}}^{2}}$

$f$ ) on retourne à $" b$ ) " jusqu'à la convergence.

Avec $\mu_{\mathrm{f}}$ ainsi trouvé, on peut calculer le tenseur $[\mu]$.

Dans les cas traités, nous avons constaté que cet algorithme converge toujours lorsque nous prenons comme valeur initiale $H_{\mathrm{f}}=0$.

4. Formulation variationnelle du problème de champ. - Le principe de la méthode variationnelle $[6,7,8]$ consiste à minimiser une certaine fonctionnelle au lieu de résoudre directement l'équation descriptive des phénomènes physiques du système en question, soit ici :

$$
\begin{aligned}
\operatorname{div} \mathbf{B} & =0 \\
\operatorname{rot} \mathbf{H} & =0 .
\end{aligned}
$$

Comme on suppose qu'il n'y a pas de courant dans le domaine, on définit un potentiel scalaire $V$ dont on dérive le champ $\mathbf{H}$ :

$$
\mathbf{H}=-\operatorname{grad} V .
$$

L'équation à résoudre est alors :

$$
\nabla \overline{\bar{\mu}} \nabla V=0
$$

où l'opérateur $\mu$ est défini par la matrice de perméabilité (cf. l'expression (14)).

Introduisons, pour un domaine $D$, la fonctionnelle :

$$
\mathcal{F}=\int_{D} F(V, \nabla V) \mathrm{d} D
$$

où la coénergie $F$ par unité de volume est telle que

$$
F=\int_{0}^{H_{x}, H_{y}} \text { B.dh . }
$$

Après calculs $F$ s'écrit aussi :

$$
F=\varepsilon \int_{0}^{\mathbf{H}_{\mathbf{a}}} \mathbf{B}_{\mathrm{a}} \mathrm{dh} \mathbf{h}_{\mathrm{a}}+(1-\varepsilon) \int_{0}^{\mathbf{H}_{\mathrm{f}}} \mathbf{B}_{\mathrm{f}} \mathrm{dh} \mathbf{h}_{\mathrm{f}}
$$

On voit donc, que la coénergie de l'ensemble $D$ a une valeur bien déterminée, égale à la somme des coénergies des matériaux qui forment le domaine $D$. Ceci prouve que cette grandeur est une fonction d'état.

On doit chercher, parmi toutes les fonctions $V$ qui satisfont les conditions de Dirichlet (potentiels imposés sur une partie de la frontière de $D$ ), celle qui rend $\mathcal{F}$ extrémale. On doit donc vérifier l'équation d'Euler relative à ce problème variationnel, soit

$$
\frac{\partial F}{\partial V}-\frac{\partial}{\partial x}\left(\frac{\partial F}{\partial V_{x}^{\prime}}\right)-\frac{\partial}{\partial y}\left(\frac{\partial F}{\partial V_{y}^{\prime}}\right)=0
$$

où $V_{x}^{\prime}$ et $V_{y}^{\prime}$ sont les dérivées partielles de $V$ telles que :

$$
V_{x}^{\prime}=\frac{\partial V}{\partial x}=-H_{x} \quad \text { et } \quad V_{y}^{\prime}=\frac{\partial V}{\partial y}=-H_{y} .
$$

En plus, la formulation variationnelle montre [5] que, sur le restant de la frontière (où $V$ n'a pas été imposé), l'équation suivante sera vérifiée

$$
\frac{\partial F}{\partial V_{x}^{\prime}} n_{x}+\frac{\partial F}{\partial V_{y}^{\prime}} n_{y}=0
$$

où

$$
\mathbf{n}=n_{x} \mathbf{i}+n_{y} \mathbf{j}
$$

est la normale à la frontière, $\mathbf{i}$ et $\mathbf{j}$ étant les vecteurs unitaires selon les axes $\mathrm{O} x$ et $\mathrm{O} y$.

$$
\text { Ainsi, étant donné que : } F=\int_{0}^{H_{x}, H_{y}} \text { B.dh est une }
$$

fonction d'état qui ne dépend donc pas du chemin d'intégration on peut écrire, en intégrant soit sur le chemin (1), soit sur le chemin (2) de la figure 3

$$
\begin{aligned}
\frac{\partial F}{\partial H_{x}}=\frac{\partial}{\partial H_{x}} & {\left[\int_{0}^{H_{x}} B_{x}\left(h_{x}, H_{y}\right) \mathrm{d} h_{x}+\right.} \\
& \left.+\int_{0}^{H_{y}} B_{y}\left(0, h_{y}\right) \mathrm{d} h_{y}\right]=B_{x}\left(H_{x}, H_{y}\right)
\end{aligned}
$$

$$
\begin{aligned}
\frac{\partial F}{\partial H_{y}}= & \frac{\partial}{\partial H_{y}}\left[\int_{0}^{H_{x}} B_{x}\left(h_{x}, 0\right) \mathrm{d} h_{x}+\right. \\
& \left.\quad+\int_{0}^{H_{y}} B_{y}\left(H_{x}, h_{y}\right) \mathrm{d} h_{y}\right]=B_{y}\left(H_{x}, H_{y}\right) .
\end{aligned}
$$

$\mathrm{Si}$ on remplace ces expressions ci-dessus dans 


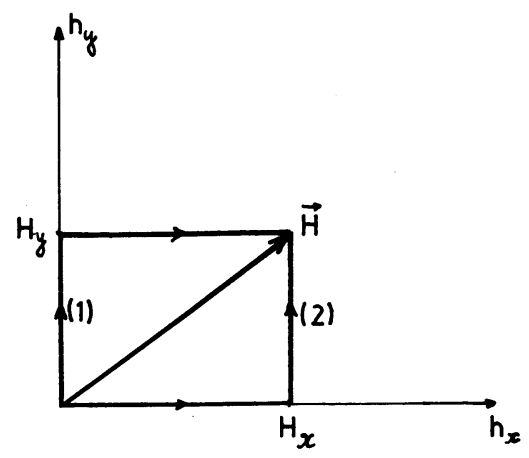

Fig. 3. - Chemins d'intégration du champ.

[Field integration ways.]

l'équation d'Euler (16) et si on tient compte de ce que $F$ ne dépend pas explicitement de $V$, il vient

$$
\frac{\partial}{\partial x}\left[B_{x}\left(H_{x}, H_{y}\right)\right]+\frac{\partial}{\partial y}\left[B_{y}\left(H_{x}, H_{y}\right)\right]=0
$$

soit l'équation $\operatorname{div} \mathbf{B}=\mathbf{0}$.

En ce qui concerne l'expression (17), on a, d'après (18) :

$$
B_{x} n_{x}+B_{y} n_{y}=0, \quad \text { soit } \mathbf{B} . \mathbf{n}=0,
$$

ce qui représente la condition de Neumann.

5. La résolution numérique. - La résolution numérique de ce problème s'effectue alors par la méthode classique des éléments finis. Notons simplement que la frontière du domaine $D$ étudiée comporte une partie où le potentiel est imposé (condition de Dirichlet) et une partie où l'on sait $a$ priori que le champ $y$ est tangent (condition de Neumann). Ce domaine est découpé en éléments à l'intérieur desquels le potentiel varie de façon linéaire : les éléments sont des triangles et éventuellement des quadrilatères qui sont alors redécoupés automatiquement en triangles. Dans chaque élément, il n'y a qu'un seul matériau : le champ magnétique $\mathbf{H} y$ est constant ainsi que la perméabilité $[\mu]$.

Si nous avons $N$ nœuds au total, $M$ nœuds où les potentiels ont été imposés, les inconnues sont alors les valeurs des potentiels $V_{\mathrm{i}}$ aux $N-M$ nœuds restants. Leur détermination se fait par une méthode itérative dont le principe est le suivant :

a) on choisit une valeur initiale pour chaque $V_{\mathrm{i}}$.

b) avec $V_{\mathrm{i}}$, on calcule $H$ dans chaque élément.

c) avec $H$, on obtient $[\mu]$ au moyen de la méthode d'approximations successives (cf. § 3 ).

d) ayant $[\mu]$, l'utilisation de la méthode variationnelle par éléments finis permet d'établir le système d'équations linéaires auxquelles obéissent les $V_{\mathrm{i}}$ (cf. annexe). On le résout par la méthode directe de Gauss.

$e$ ) avec les nouveaux $V_{\mathrm{i}}$, on revient en $b$ ) jusqu'à ce que la méthode converge.
Cet algorithme est le même que celui du problème isotrope, sauf qu'au pas $c$ ), le calcul de $\mu$ s'effectue directement.

6. Tests de convergence et résultats obtenus. Nous décrivons maintenant un des tests effectués afin d'observer la convergence de cette méthode.

Nous avons, sur la figure 4 , deux plots constitués par des feuilletages. Le plot supérieur est feuilleté verticalement, tandis que l'autre l'est horizontalement. Le coefficient de remplissage est de 0,85 . On impose une DDP magnétique entre le bord supérieur et le bord inférieur. Sur les bords latéraux, on a la condition de Neumann, c'est-à-dire que les lignes d'induction leur sont parallèles. Les courbes caractéristiques du fer sont celles de la figure 5 .

Nous avons testé la convergence du potentiel sur les nœuds numérotés $37,49,72,93$ sur la figure 4 . Nous avons fait le calcul pour plusieurs valeurs de DDP

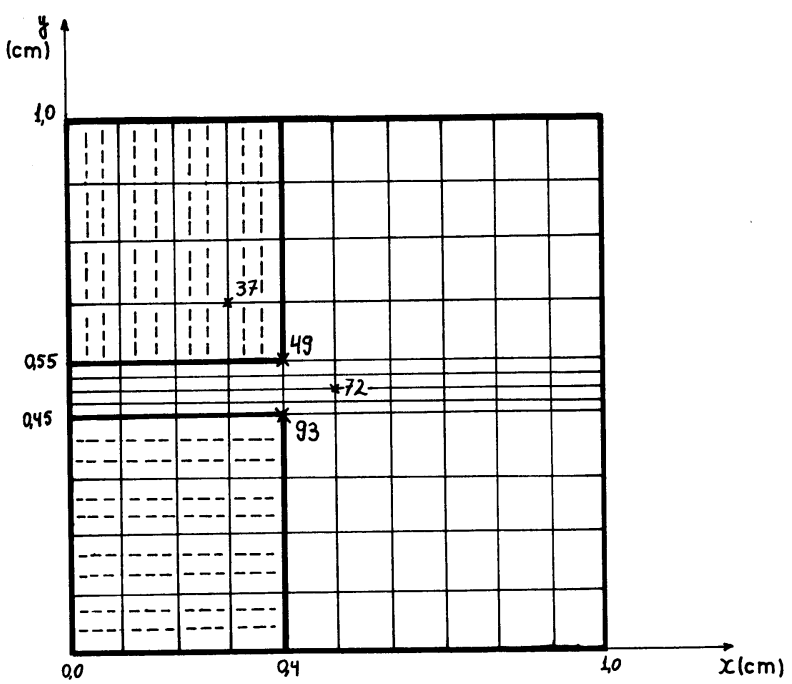

Fig. 4. - Maillage d'essai.

[Test finite elements subdivision.]

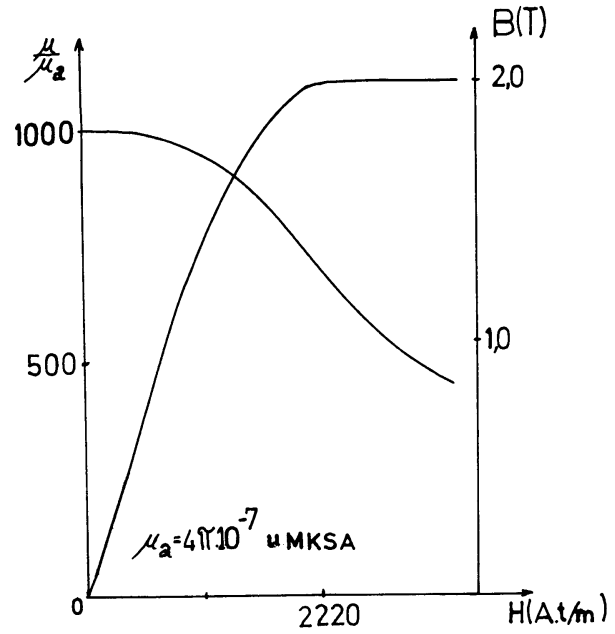

Fig. 5. - Courbes caractéristiques du fer.

[Iron caracteristic curves.] 
magnétique. Nous montrons sur la figure 6, en fonction du nombre d'itérations, les résultats correspondant à une DDP appliquée égale à 2330 At qui amène le fer à travailler dans la zone du coude de saturation. Cette valeur de DDP représente un cas délicat de convergence (on remarquera que la convergence, dans d'autres cas où le fer n'est pas encore saturé, est obtenue dès la deuxième itération alors que lorsqu'il est complètement saturé, celle-ci est obtenue dans la cinquième itération). La nature de ce problème impose comme condition nécessaire que les flux à travers toute ligne horizontale soient égaux. Nous avons donc calculé les flux à travers diverses lignes et nous avons trouvé (DDP $=2330 \mathrm{~A} . \mathrm{t})$ qu'à la $15^{\mathrm{e}}$ itération l'erreur relative entre les valeurs extrêmes n'excède pas $5 \%$ et est inférieure à $2 \%$ à la vingtième.

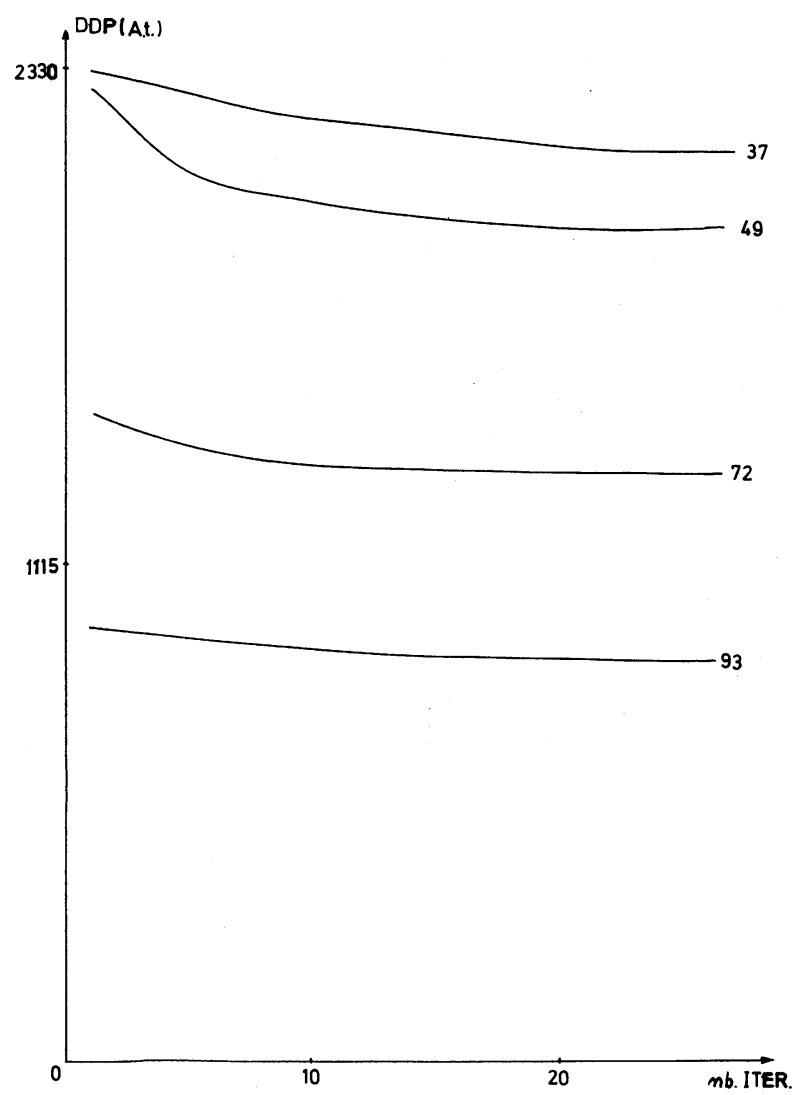

Fig. 6. - Convergence du potentiel aux nœuds.

[Ventices potential convergence.]

Sur les figures $7 a$ et $7 b$ nous donnons, pour les mêmes conditions de feuilletage définies sur la figure 4, deux tracés de lignes d'induction obtenus à l'aide d'un traceur connecté à l'ordinateur. Ces cartes correspondent à une faible DDP, donc à un régime linéaire. On remarque la canalisation du flux vers les bores latéraux

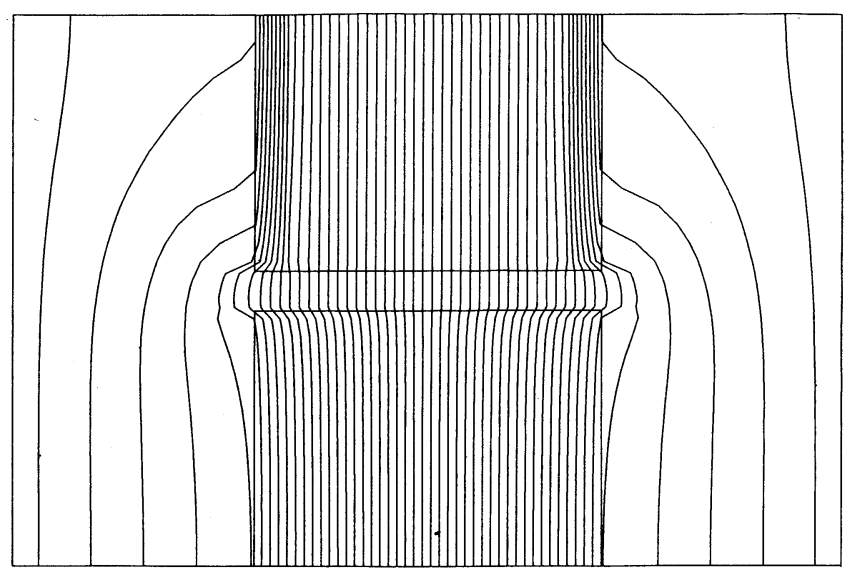

FEUILLETAGE MIXTE REMP $=85 / 100$

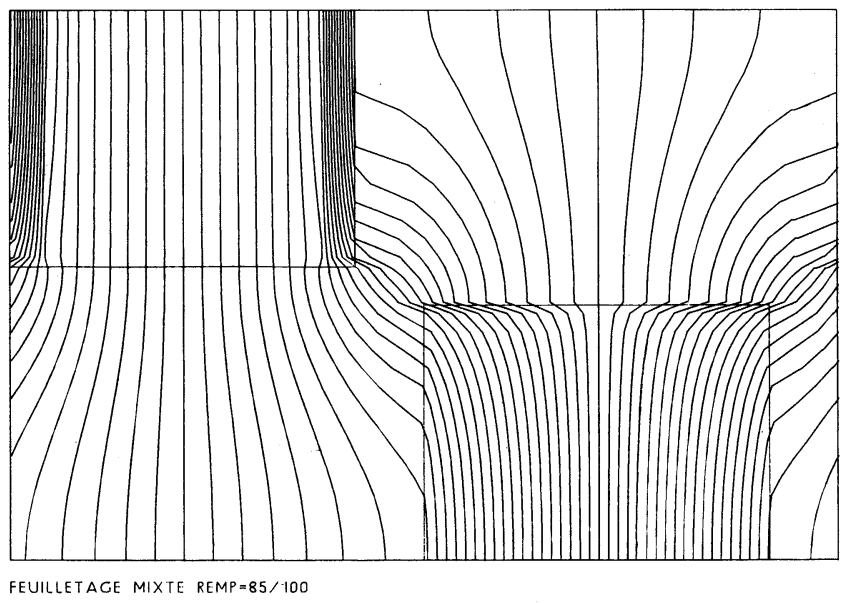

Fig. $7 a, b .-$ Distribution du flux magnétique. [Magnetic flux distribution.]

du plot supérieur, ce qui est dû, évidemment, au feuilletage vertical de ce plot.

7. Conclusion. - La méthode décrite a fourni, au cours des exploitations faites, des résultats satisfaisants, aussi bien en régime linéaire qu'en régime saturé.

Nous avons décrit ici uniquement les principes de la méthode, sans évoquer les aspects de programmation informatique correspondants. Signalons, que le programme ainsi mis au point est rapide et que la méthode itérative introduite au niveau du calcul de la perméabilité du feuilletage ne ralentit que très peu le calcul global, par rapport au cas isotrope.

Remarquons finalement que l'application de la méthode variationnelle au problème anisotrope, ici décrite, ne se limite pas uniquement au cas d'un feuilletage, mais est valable chaque fois que l'on peut définir un tenseur $[\mu]$ de perméabilité. 


\section{Bibliographie}

[1] Pakdel, Quichaud, Robert, Etude d'un moteur à réluctance variable à feuilletage axial. Revue Phys. Appl. 15 (1980) 331-339.

[2] Wexter, A., Finite element field analysis of an inhomogeneous anisotropic reluctance rotor. IEEE Trans. (1973), p. 15.

[3] Bastos, J. P., Goyet, R., Lucidarme, J., Performances intrinsèques des machines à réluctance variable à disques imbriqués. Revue Phys. Appl. 15 (1980) 45.

[4] Rioux, C., Théorie des machines comportant des matériaux ferromagnétiques. Publication interne LF no 70 (1977) bât. 214,91405 Orsay.

[5] Bastos, J. P., Calcul des performances intrinsèques des machines à réluctance variable polydiscoïdes par une méthode d'éléments finis. Thèse de docteur-ingénieur (soutenue 5/1980).

[6] Silvester, Chari, Finite element solution of saturable magnetic field problems. IEEE Trans. Power PAS/89 (1970) no 7.
[7] Norrie, D. H., Devries, G., An introduction to finite elements analysis (Academic Press, New York, San Francisco, London).

[8] ZIENKIEWICs, The finite elements method in engineering science (McGraw-Hill).

[9] Rioux, C., Théorie générale comparative des machines électriques établie à partir des équations du champ électromagnétique. Revue Gén. Electr. 79 (1970) 415.

[10] Jablon, Tournaire, Comparaison des diverses modélisations numériques d'un système magnétique saturable. Revue Gén. Electr. 84 (1975) 9.

[11] WeXLER, A., Some recent developments in field calculation. IEEE Trans. on magnetics MAG 15N (1979).

[12] WeGGleR, P. T., Intermag (1976) p. 168.

[13] Kasuga, T., Takasaki, E., Ando, A., Kihara, M. et Endo, K., Intermag (1976) p. 177.

\section{Annexe}

Discrétisation de la formulation variationnelle. Pour notre cas, avec les notations classiques de [6], on a, pour le potentiel dans un triangle :

$$
V(x, y)=\sum_{l=1,2,3} \frac{1}{D}\left(p_{l}+q_{l} x+r_{l} y\right) V_{l}
$$

où $D$ est le double de la surface du triangle.

On obtient, alors, dans un triangle $t_{\mathrm{i}}$ :

$$
\left[H_{\mathrm{i}}\right]=-\frac{1}{D_{\mathrm{i}}}\left[\begin{array}{lll}
q_{1} & q_{2} & q_{3} \\
r_{1} & r_{2} & r_{3}
\end{array}\right]\left[\begin{array}{l}
V_{1} \\
V_{2} \\
V_{3}
\end{array}\right] .
$$

On a aussi, en dérivant par rapport à un nœud $K$

$$
\left[\frac{\partial H_{\mathrm{i}}}{\partial V_{\mathrm{K}}}\right]=-\frac{1}{D_{\mathrm{i}}}\left[\begin{array}{l}
q_{\mathrm{K}} \\
r_{\mathrm{K}}
\end{array}\right] \text {. }
$$

En appliquant la méthode variationnelle on a :

$$
\frac{\partial \mathcal{F}_{\mathbf{i}}}{\partial V_{\mathbf{K}}}=\frac{\partial \mathcal{F}_{\mathrm{i}}}{\partial H_{x}} \cdot \frac{\partial H_{x}}{\partial V_{\mathbf{K}}}+\frac{\partial \mathcal{F}_{\mathbf{i}}}{\partial H_{y}} \cdot \frac{\partial H_{y}}{\partial V_{\mathbf{K}}} .
$$

Avec les expressions $(18 a, b)$, après des manipulations algébriques; il vient :

$$
\frac{\partial \mathcal{F}_{\mathbf{i}}}{\partial V_{\mathbf{K}}}=\mathbf{B}_{\mathbf{i}} \cdot \frac{\partial \mathbf{H}_{\mathbf{i}}}{\partial V_{\mathbf{K}}} \cdot \frac{D_{\mathbf{i}}}{2}
$$

qui en utilisant les expressions (19) et (20), se met sous la forme matricielle suivante :

$$
\begin{aligned}
{\left[\begin{array}{l}
\frac{\partial \mathcal{F}_{\mathrm{i}}}{\partial V_{1}} \\
\frac{\partial \mathcal{F}_{\mathrm{i}}}{\partial V_{2}} \\
\frac{\partial \mathcal{F}_{\mathrm{i}}}{\partial V_{3}}
\end{array}\right]=\frac{1}{2 D_{\mathrm{i}}}\left[\begin{array}{ll}
q_{1} & r_{1} \\
q_{2} & r_{2} \\
q_{3} & r_{3}
\end{array}\right]\left[\begin{array}{ll}
\mu_{x} & \mu_{x y} \\
\mu_{y x} & \mu_{y}
\end{array}\right] \times } \\
\times\left[\begin{array}{lll}
q_{1} & q_{2} & q_{3} \\
r_{1} & r_{2} & r_{3}
\end{array}\right]\left[\begin{array}{c}
V_{1} \\
V_{2} \\
V_{3}
\end{array}\right] .
\end{aligned}
$$

Toutes ces matrices (21) des triangles sont assemblées dans un système d'équations linéaires [5] qui est résolu après insertion des conditions aux limites de Dirichlet. 